\title{
An Ancient Enzyme Finds a New Home: Prevalence and Neofunctionalization of Trypsin in Marine Phytoplankton
}

\section{Yanchun You}

College of Ocean and Earth Sciences, Xiamen University,

\section{Xueqiong Sun}

College of Ocean and Earth Sciences, Xiamen University,

Senjie Lin ( $\nabla$ senjie.lin@uconn.edu )

University of Connecticut https://orcid.org/0000-0001-8831-6111

\section{Article}

Keywords: trypsin, phylogenetics, tandem duplication, evolution, environmental stimuli

Posted Date: November 16th, 2021

DOI: https://doi.org/10.21203/rs.3.rs-1008417/v1

License: (c) (i) This work is licensed under a Creative Commons Attribution 4.0 International License.

Read Full License 


\title{
An ancient enzyme finds a new home: prevalence and neofunctionalization of trypsin in marine phytoplankton
}

\author{
Yanchun You ${ }^{1}$, Xueqiong Sun ${ }^{1}$, Senjie Lin ${ }^{1,2,3^{*}}$
}

Yanchun You: fjyhy@163.com

Xueqiong Sun:22320201151289@stu.xmu.edu.cn

Senjie Lin: senjie.lin@uconn.edu

${ }^{1}$ State Key Laboratory of Marine Environmental Science, College of Ocean and Earth Sciences, Xiamen University, Xiamen 361005, China.

${ }^{2}$ Laboratory of Marine Biology and Biotechnology, Qingdao National Laboratory of Marine Science and Technology, Qingdao 266237, China.

${ }^{3}$ Department of Marine Sciences, University of Connecticut, Groton, CT, United States.

*Correspondence: Senjie Lin: senjie.lin@uconn.edu

\begin{abstract}
Trypsin is an ancient protease best known as a digestive enzyme in animals, and traditionally believed to be absent in plants and protists. Here, we surveyed the distribution, diversity, evolution and potential functions of trypsin genes in global ocean phytoplankton, the major primary producers in the aquatic ecosystem. Our analysis indicates that trypsin genes are widely distributed both taxonomically and geographically in marine phytoplankton. Furthermore, by systematic comparative analyses we documented lineage-specific diversity and expansion of trypsin genes in the evolution of marine phytoplankton. Genome-wide analyses revealed that trypsin genes were more prevalent in diatoms than in other lineages. Moreover, the expression
\end{abstract}


of trypsin genes in diatom tended to be more responsive to environmental stimuli. The duplication and neofunctionalization of trypsin genes may be important in diatoms to adapt to dynamical environmental conditions, contributing to diatoms' dominance in the coastal oceans. This work advances our knowledge on the distributions and neofunctionalizations of this ancient enzyme and creates a new research direction in the phytoplankton biology.

Keywords: trypsin, phylogenetics, tandem duplication, evolution, environmental stimuli 


\section{Introduction}

2 Trypsin (EC 3.4.21.4) is known as pancreatic serine proteolytic enzyme, which specifically

3 cleaves the carboxyl end of the lysine and arginine residues in polypeptides. First discovered

4 almost 150 years ago ${ }^{1}$, trypsin is arguably the first enzyme known to science and the best studied

5 protein, and probably is the best exploited enzyme for protein biotechnology. Yet beyond the

6 knowledge of it as a digestive enzyme, understanding of its occurrence and function across the

7 tree of life is very limited.

8 Trypsin represents a conserved family of enzymes occurring in organisms ranging from bacteria

9 to mammals ${ }^{2,3}$. It is believed to be absent in plants and protists ${ }^{4,5}$. Indeed, many animal trypsin

10 genes have extremely well-understood physiological roles, to digest protein food and to activate

11 zymogen, an activator of other proteases ${ }^{6,7}$, etc.. In the case of fish, the expression of trypsin is

12 affected by influences protein digestion rate, amino acid absorption and transport, protein

13 synthesis, and growth rate ${ }^{2,8,9}$. However, the gene characteristics, phylogenetic perspectives and

14 function of trypsin in phytoplankton are unexplored and enigmatic.

In the current MEROPS database, there are 109 trypsin enzymes, all of which are exclusively from animals. In a recent metatranscriptomic study on a phytoplankton community, diatom trypsin genes were found to be highly expressed, accounting for $1 \%$ of the total diatom transcriptome when diatoms were dominant ${ }^{10}$. This raises questions as to whether trypsin occurs broadly, how it has evolved, and what roles it plays, in phytoplankton, which represent the majority of plantae diversity and 50\% of global carbon fixation and oxygen production. Here, we analyzed the Tara Oceans metatranscriptomic dataset to detect occurrence and quantify expression of trypsin genes in phytoplankton in the global ocean and their relationship with environmental factors. We further documented sequence diversity, lineage-specific expansion and phylogenetic perspectives of trypsin genes by mining existing algal genome data and analyzed gene structure, evolutionary characteristics, and expression patterns from nine species that represent major phyla of algae.

\section{Results}



plankton

To understand how broadly trypsin occurs in marine plankton, we mined existing data. Using Hidden Markov Models, we aligned trypsin (PF00089) and trypsin-like (PF13365) conserved domains against the MATOU databases. Trypsin and trypsin-like homologous genes were presented in all global sampling sites in the Tara Oceans expedition with 129,512 and 6,167 hits, respectively. Interestingly, trypsin sequences were found predominantly in the surface water layer (SRF) and, less abundantly in the deep chlorophyll maximum layer (DCM), and both were more abundant in the larger sized organisms (180-2000 $\mu \mathrm{m})($ Fig. 1a). The trypsinlike sequences show similar distribution with trypsin with respect to latitudes and the sampled depths, but were more abundant in the smaller sized organisms (0.8-5 $\mu \mathrm{m})$ (Fig. 1b).

Taxonomically, $96 \%$ of the trypsin hits was originated from metazoans, and a small percentage from dinoflagellates $(2.0 \%)$, haptophytes $(0.2 \%)$, diatoms $(0.1 \%)$, and cryptophytes $(0.1 \%)$ (Fig. 1c). For trypsin-like, in contrast, $41 \%$ of the hits were from haptophytes, $20 \%$ from stramenopiles (dominantly Pelagophyceae), 12\% from dinoflagellates, $8 \%$ from chlorophytes, and only $6 \%$ from metazoans (Fig. 1d). Therefore, trypsin and trypsin-like genes are widely distributed in marine plankton both taxonomically and geographically, but are dominantly in Arthropoda and phytoplankton, respectively.

\section{Abundance and evolutionary dynamics of trypsin genes in algal genomes}

To better understand the lineage-specific diversity and expansion of trypsin during phytoplankton evolution, we searched for trypsin sequences from available sequenced genomes across four major phyla of algae, including Rhodophyta (C. merolae), Chlorophyta $(C$. reinhardtii), Dinophyta (F. kawagutii) and Bacillariophyta (T. pseudonana, P. tricornutum, T. oceanica, F. solaris, F. cylindrus and P. multiseries). For comparison, the genome of the land plant model $A$. thaliana was also analyzed. A total of 291 algal trypsin genes and 16 A. thaliana trypsin genes were identified and named based on their species name abbreviations and chromosomal locations (supplementary table 1). The number of trypsin genes varies in 
examined algal genome from 5 to 65 (Fig. 2), accounting for $0.93 \%$ to $5.68 \%$ of their predicted proteomes.

To explore the evolutionary trend of trypsin, we inferred a species phylogenetic tree based on the $\mathrm{NCBI}$ Taxonomy Common Tree (https://www.ncbi.nlm.nih.gov/Taxonomy/CommonTree/wwwcmt.cgi) database and mapped the genome size, coding gene number, and the number of identified trypsin genes on the tree (Fig. 2). The strongly supported tree suggest that ancestral algae, dated prior to the C. merolae, likely already contained a repertoire of trypsin genes. Based on the tree, it appears that diatoms have undergone a greater trypsin gene family expansion than dinoflagellates, which is disproportionate to their genome sizes (Fig. 2). However, the numbers of trypsin genes vary considerably across alga lineages, even between diatom species, showing no phylogenetic trends. For instance, P. tricornutum and F. solaris are phylogenetically closely related, both belonging to the order of Naviculales, similar in genome size, but are very different in trypsin gene copy number (10 and 65 copies, respectively), indicating a trypsin gene expansion hotspot in F. solaris.

The trypsin gene also varies significantly between lineages in chromosomal locations, open reading frame lengths, amino acid numbers, molecular weights and isoelectric points (pIs) (supplementary table 1). Large differences can even be found between different members of the same lineage. For example, for PtTryp alone, the length ranges from 366 (PtTryp3) to 726 amino acids (PtTryp8), and the molecular weights from $39.66 \mathrm{kDa}$ (PtTryp5) to $76.63 \mathrm{kDa}$ (PtTryp8).

\section{Classification of trypsin genes into two classic subfamilies}

To analyze the evolutionary relationships of trypsin genes among the nine selected algae, an unrooted phylogenetic tree was constructed using their conserved amino acid sequences (Fig. 3). The 291 trypsin genes were well separated into two distinct subfamilies: trypsin (162 genes, Fig. 3, clade I) and trypsin-like (129 genes, Fig. 3, clade II). Most of the trypsin genes are 
81 originated from diatoms and dinoflagellates. By contrast, trypsin-like genes are more diverse, including sequences from all the selected nine species of algae.

83 Remarkably, some trypsin members were more closely related to those in the same subfamily 84 from different species than to the other trypsin genes from the same species, indicative of ancestral gene duplication and subsequent divergence. By contrast, some trypsin members were more closely related to those from the same species than to the other trypsin genes from different species, indicating recently gene duplication evens within species. Within a subfamily, most trypsin genes were clustered by phylum, suggesting a lineage-specific expansion within the trypsin or trypsin-like subfamily in algae. In C. merolae, an ancient taxon of rhodophytes, the phylum from which all non-green algae are believed to have emerged, all of its trypsin genes appeared to belong to the trypsin-like subfamily. These results indicate that trypsin genes might have arisen from trypsin-like genes.

\section{Trypsin gene clusters and evidence of duplications and differential losses}

We found that trypsin genes are not randomly distributed on different chromosomes, scaffolds or contigs, and some algal trypsin genes exist as gene clusters (Extended Data Fig. 1). Based on current genome assembly level, four of the nine alga genomes we examined contain trypsin genes organized in clusters, as shown in extended data figure 1, C. reinhardtii with 5 clusters, F. cylindrus with 4 clusters, F. solaris with 5 clusters, and T. pseudona with 4 clusters, respectively. In sum, the algae with gene clusters appear to host more trypsin genes, which might have resulted from gene duplication. Considering that the genome assembly of some algae were only at contig level, thus, some trypsin gene clusters may be missing, further gene duplication events were analyzed. For two proteins to belong to the duplicated gene pairs, two criteria must be met: (1) length of alignable sequence covers $>75 \%$ of the longer gene, and (2) the similarity of aligned regions is $>75 \%{ }^{11}$. Based on these criteria, three species, C. reinhardtii, $T$. oceanica and F. solaris, had three genes in three duplicated gene pairs out the genome collinearity blocks, 10 genes in 6 duplicated gene pairs out the genome collinearity blocks, and 34 genes in 24 duplicated gene pairs in collinearity blocks, respectively (Fig. 4; Extended Data Table 1). To examine the evolutionary constraints acting on trypsin gene family, the 
nonsynonymous/synonymous substitution ratio $(\mathrm{Ka} / \mathrm{Ks})$ was calculated for the duplicated

110 trypsin gene pairs. The majority of the duplicated trypsin gene pairs exhibited a $K a / K s$ ratio <

1111 (Extended Data Table 1), indicative of purifying selection after duplication. These results

112 indicated that some trypsin genes might have arisen by segmental chromosome duplication.

113 Of the 304 trypsin genes found from the ten species, all but 70 genes were clustered in

114 orthologous groups or paralogous groups by OrthoFinder analysis. From the phylogenetic tree

115 of 234 orthologous or paralogous trypsin genes, nine clades emerged (Fig. 5). All the species

116 included in the analysis contained a rich repertoire of trypsin genes of clade I, and its late

117 diverging position indicates that this is the most recent emergent of the trypsin family. The other

118 eight clades, however, are completely absent in C. merolae and variably occur across different

119 species. These indicate that trypsin has experienced multiple gene duplication events and

120 differential losses. In the course of evolution, C. merolae lost all paralogs from clades II through

121 IX, diatoms retained most of the trypsin of clade II, VII, VIII and IX, and the other species are

122 in between the two extremes. The remarkable sequence divergence in the more basal clades

123 (e.g. IX) suggests neofunctionalization might have occurred.

\section{Conserved and diverged trypsin gene structure}

125 To further explore the potential functions of algal trypsin genes, the conserved motifs and 126 domains of trypsin proteins were identified by MEME and hmmscan. As showed in figure 3, 12710 conserved motifs and three conserved domains were identified, and with subclade-regular 128 composition and distribution patterns. Based on the comprehensive analyses from Pfam, CDD and SMART database, motifs 2, 3, 6, 8 and 9 were responsible for the region of the conserved

130 trypsin, and motifs 5 and 10 belong to the trypsin-like serine protease, motifs 1,4 and 7 were 131 unknown, but they share similar sequence with PPSP repeat (Fig. 3; detail consensus amino 132 acid sequence shown in Extended Data Fig. 2 and Extended Data Table 2). Furthermore, the 133 three most conserved domains were trypsin, trypsin-like and PDZ-like domain, and some trypsin-like domain tend to couple with PDZ-like domain. The PDZ domain is believed to target signaling molecules to sub-membranous sites and occurs in diverse signaling proteins. As illustrated in figure 3 , the two clades of trypsin genes show diverged motif and domain 
137 compositions and distributions, but with similar patterns among the closely branches, indicating 138 that trypsin and trypsin-like genes may play different roles in cells. There are three different 139 domain composition and distribution patterns, that is, trypsin domains were only found in 140 trypsin genes, trypsin-like domains solely or couple with PDZ domains in trypsin-like genes.

141 Furthermore, a close relationship was found between the phylogenetic affiliation, conserved 142 domain and motif composition and distribution patterns, indicating that they were highly 143 conserved, which also supports the close evolutionary relationship of these members.

144 Trypsin expression profiles in different plankton groups and nutrient conditions in the 145 global ocean

146 To further understand the potential ecological functions of the ubiquitous plankton trypsin 147 genes, we investigated the relationship between trypsin gene expressions and environmental 148 factors. Interestingly, the majority (74.93\%) of trypsin transcripts were found in the larger size 149 fractions (32.31\% and $42.62 \%$ in $20-180 \mu \mathrm{m}$ and $180-2000 \mu \mathrm{m}$, respectively, $48.89 \%$ and $26.04 \%$ 150 in SRF and DCM, respectively) (Fig. 6a). By contrast, the majority (62.64\%) of trypsin-like 151 transcripts were found in the smaller size fraction $(0.8-5 \mu \mathrm{m}, 41.97 \%$ and $20.67 \%$ in SRF and DCM, respectively) (Fig. 6b). According to Malviya et al. (2016), Tara Oceans smaller size

153 fractions $(0.8-5$ and 5-20 $\mu \mathrm{m})$ are mostly composed of small diatoms and single cells. Overall, 154 both trypsin subfamilies were more abundantly expressed at surface than that at the DCM. The 155 different size-class distributions of trypsin and trypsin-like suggest that they may have different 156 functions. Furthermore, as showed in figure 6, the trypsin subfamily showed no correlation with ambient nutrient abundances except for SRF_5-20 and SRF_0.8-5 with iron and SRF_0.8-5

158 with nitrite. In contrast, the trypsin-like subfamily was closely correlated with ambient nutrient 159 conditions, especially for the medium size fractions $(5-180 \mu \mathrm{m})($ Fig. $6 \mathrm{~b})$. Moreover, the mRNA 160 abundance of trypsin-like were significant positively correlated with ambient nutrient changes, 161 while showed anticorrelated with $\mathrm{Fe}$ and $\mathrm{Si}$. With trypsin, only the mRNA abundance of 162 SRF_0.8-5 $\mu \mathrm{m}$ showed anticorrelation with $\mathrm{Fe}$ and positive correlation with $\mathrm{NO}_{2}$ 5m, while 163 SRF_5-20 $\mu \mathrm{m}$ was positive correlated with Fe. Moreover, $\mathrm{NO}_{2}, \mathrm{NO}_{3} \_5 \mathrm{~m}$ and $\mathrm{NO}_{3} \mathrm{NO}_{2}$ could 164 modulated more size-class of trypsin-like expression. 
167 To gain a deeper insight into the functions of the trypsin genes analyzed, we took advantage of currently available diatom EST libraries and Symbiodiniceae and Algal Genomic Resource (SAGER), which included 16 transcriptomic libraries in P. tricornutum, seven in T. pseudonana, and six in F. kawagutii, each derived from cells grown under different conditions ${ }^{12,13}$. After normalizing trypsin gene expression for the diatoms and dinoflagellates, a hierarchical clustering ${ }^{14}$ of the P. tricornutum, T. pseudonana and F. kawagutii trypsin genes was made to identify groups of genes with similar expression patterns and the libraries with similar gene expression profiles. The expression patterns of dinoflagellate trypsin genes exhibited constitutive expression under different growth conditions, while the expression of diatom trypsin genes tended to be more dynamic, and exhibited gene-differential and growth conditionspecific patterns (Fig. 7). In diatoms, the expression of trypsin genes showed responses to growth conditions (Fig. 7a), with more genes responding to more specific conditions. For example, in P. tricornutum, trypsin genes from clade I responded to fewer growth conditions than those from clade II. Notably, PtTryp 2 was the only gene showing differential expression across all these 16 different growth conditions with a higher expression (Fig. 7a), indicating that the gene might play important roles in responding to various environmental stresses. In $T$. pseudonana, only half of the 36 identified trypsin genes were expressed under the seven different conditions, most of the other 18 genes are organized in tandem repeats, which are likely to be pseudogenes. Similarly, in T. pseudonana clusters of trypsin genes represent more condition-specific expression pattern, genes of clade I and II were less responsive, while genes of clade III were more responsive (Fig. 7b). TpTryp14 was significantly more highly expressed in all T. pseudonana libraries but in the 'temperature limited' (TL) library. T. pseudonana trypsin genes appeared to be more strongly responsive to the nitrate plus, while in temperature

190 limited was just the opposite. Most F. kawagutii trypsin genes, except FkTryp14 and FkTryp13, 191 were found to be consistently expressed throughout different growth conditions (Fig. 7c), those 192 highly and moderately expressed genes (clade I and II) may function as housekeeping genes 193 involved in basic physiological processes. All these results showed that algal trypsin genes with 
194 diverse and complicated expression patterns, suggesting their diversified functions and may

195 function as biological interactions or cross-talk between in response to particular stimuli.

\section{Discussions}

\section{Widespread occurrence of trypsin in marine phytoplankton}

198 In many eukaryotic taxonomic groups, especially in animals, trypsin gene family has undergone

199 repeated cycles of duplication and divergence to perform a wide spectrum of physiological 200 activities $^{15-21}$, but were previously believed to be absent in plants and protists ${ }^{4}$. With the advances of sequencing technology, increasing plant and protist genomes have been sequenced,

202 facilitating detection of trypsin genes. However, most of the sequences from plants or protists 203 that contain the trypsin or trypsin-like domains are usually recognized as unknown function 204 proteins, and much less data is available for marine phytoplankton trypsin. Our report here is a systematic documentation of trypsin and trypsin-like genes in marine phytoplankton. We identified 291 trypsin genes across four major phyla of algae that had sequenced genomes

207 (Bacillariophyta, Dinophyta, Cyanophyta, and Chlorophyta). In addition, we identified 129,512 and 6,167 putative trypsin and trypsin-like genes from Tara Oceans metatranscriptomic

209 datasets, indicating wide taxonomic and geographic distribution of trypsin and trypsin-like 210 genes in the global ocean.

211 Extensive duplication and evolutionarily conserved structure of trypsin in marine 212 phytoplankton

213 Expansion of gene families tend to emerge new biological functions, which with the relatively

214 high rate of being duplicated or retained ${ }^{22}$. Our analysis results show high copy numbers of 215 trypsin and trypsin-like genes occur in some algal lineages, suggesting the functional 216 importance of these genes. Their high copy numbers indicate that rampant gene duplication 217 might have occurred during evolution. This is supported by multiple lines of evidence. First, 218 compared with known species, the number of trypsin genes in phytoplankton tends to account 219 for a larger proportion of the total number of genes in the whole genome, especially for diatoms. 220 Second, the topology of phylogenetic tree suggests multiple duplication events during algal 
evolutions, especially in diatoms (Fig. 3). Third, repetitive trypsin genes cluster in tandem repeats or otherwise in pairs or in apparently duplicated segments, indicating that trypsin gene duplication is common in marine phytoplankton (Fig. 4). Last, a mass of identified trypsin genes (76.97\%) from ten sequenced algal genomes were clustered in orthologous groups or paralogous groups (Fig. 5). Similarly, trypsin gene duplications have occurred in human, mosquito, Drosophila melanogaster and Plutella xylostella genome sequences ${ }^{17,23-25}$. These examples suggest that local duplication events by unequal crossing-over as well as segmental duplication have contributed to the expansion of this gene family in marine genomes. Furthermore, the orthologous and paralogous gene analysis, combined with phylogenetic trees, indicate that complex pattern of differential losses and duplications occurred to trypsin genes during the evolution of marine phytoplankton, creating the highly variable gene copy numbers between algal species. The expansion of trypsin gene families in some diatoms may confer adaptive advantage, and in those cases, neofunctionalization might be involved.

234 A close relationship was found between the phylogenetic affiliation, conserved domain and motif composition and distribution patterns, indicating that they were highly conserved (Fig. 3). The conserved motif and domain structure of trypsin proteins, especially those that are responsible for catalytic triad and substrate-binding pocket, is crucial for their function ${ }^{26}$. Moreover, the two clades of trypsin genes showed diverging motif and domain compositions and distributions. The clade I trypsin genes tend to only contain the N-terminus trypsin domain, and mainly consist of trypsin motifs, while some of the clade II trypsin-like genes only have Nterminus trypsin-like domain that consist of trypsin and trypsin-like motifs, and some genes are additionally coupled with PDZ domain.

\section{Potential roles of phytoplankton trypsin in response to environmental stress}

244 Trypsin is known to function in peptide hydrolysis, protein cleavage, and degradation in various physiological processes in both vertebrates and invertebrates, e.g. coagulation, clot resolution, digestion, fertilization, blood pressure regulation, tissue development and homeostasis, and immunity ${ }^{27}$. Moreover, in $A$. thaliana, we found that 16 Deg/HtrA protease family genes, which have been shown to function in maintaining protein homeostasis and protein processing ${ }^{28,29}$, 
contain trypsin or trypsin-like domains. Nothing is known about the function of trypsin and trypsin-like proteins in phytoplankton, but the large amount of gene duplication documented in the present study suggests that they may have an important role in phytoplankton. This is further supported by the strikingly high expression of diatom trypsins in a diatom-dominated natural plankton assemblage ${ }^{30}$.

We hypothesize that like trypsin in fish and other animals ${ }^{2,8,31}$, phytoplankton trypsin may be involved in important cell processes and responses to various environmental factors. Based on this hypothesis, it is expected that trypsin expression would be modulated by environmental factors. Our in-depth analysis on Tara Oceans global eukaryotic metatranscriptome ${ }^{32}$, diatom EST Database ${ }^{12}$ and SAGER Database ${ }^{33}$ indicates significant correlations of trypsin and trypsin-like expressions with environmental factors. With the support from the various existing datasets, our hypothesis about the importance of the ancient enzyme in phytoplankton stands for rigorous and specific examination in future research.

\section{Conclusions}

263 Despite being a classic textbook example and a well-described group of enzymes, trypsin in marine phytoplankton is poorly understood and has been hardly explored. In this study, a wide taxonomic and geographic distribution of trypsin and trypsin-like genes was found, illuminating the potential importance of the trypsin-domain function in phytoplankton in the global ocean. We conducted integrative analyses on the trypsin gene structure, lineage-specific duplication, phylogenetic perspectives and expression modulation of trypsin family in algae. The differences in diverse duplication and expression of the trypsin gene family across different species hint at a key role. Our results suggest that major steps in the evolution of the gene family reflected key events triggering diatom radiation and diversification. Diatoms are one of the most abundant and diverse groups of marine phytoplankton, respond rapidly to the supply of new nutrients, often out-competing other phytoplankton. The more gene expansion, diversified features and expression patterns of the trypsin across different diatom species are inferred to be associated with the capacity of diatom to out-competing other phytoplankton. The conservation of subclades, lineage-specific duplication, the high homolog retention rate and the conservation 
277 of expression patterns among different algae underlie the high biological importance of the

278 trypsin gene family in general and of distinct subclades in particular across marine

279 phytoplankton. This study represents a systematic and comprehensive study on genome-wide 280 identification, lineage-specific duplication, phylogenetic perspectives, gene characterization

281 and expression profiling of the trypsin family in phytoplankton. Gene duplication and sequence 282 divergence are helpful for in-depth exploration of the evolution of novel gene functions, and 283 our findings provide a base for functional research on specific trypsin genes, a better 284 understanding of the evolution of phytoplankton trypsin.

\section{Materials and Methods}

\section{Detection of trypsin genes in the Tara Oceans datasets}

287 Extensive search for putative trypsin and trypsin-like genes was performed in both the Tara 288 Oceans eukaryote unigene catalog and metatranscriptomes (MATOUv1+metaT) ${ }^{32,34}$, using the 289 trypsin (Pfam ID: PF00089) and trypsin-like (Pfam ID: PF13365) domain based on profile 290 Hidden Markov Models (HMM), with an E-value $\leq 1.0 \mathrm{E}^{-10}$. MATOU is a catalog of 116 million 291 unigenes obtained from poly-A cDNA sequencing for samples of different size fractions and 292 different water layers, available at the OGA website (http://tara293 oceans.mio.osupytheas.fr/ocean-gene-atlas/).

\section{Identification of trypsin genes from sequenced algal genomes}

295 Whole genome sequences were downloaded from the Ensembl Protists Database 296 (http://protists.ensembl.org) and the NCBI Genome Database

297 (https://www.ncbi.nlm.nih.gov/genome). To identify trypsin genes, the hmmsearch analysis 298 was conducted. We downloaded the HMM profile of trypsin and trypsin-like (PF00089 and 299 PF13365) from Pfam protein family database (http:// pfam.xfam.org/) and used it as the query $300 \quad(\mathrm{P}<0.001)$ by hmmsearch from the whole genome protein sequences. Top hits of the search 301 were selected to build the species-specific HMM for the 2nd round of hmmsearch to yield all 302 trypsin genes from the selected genome. To avoid missing probable trypsin members because 303 of incomplete trypsin domains, a BLASTP-algorithm based search was conducted using trypsin 
304 amino acid sequences from NCBI and UniProt database as queries with an e-value $\leq 1 \mathrm{e}-5$ as the

305 threshold. After removing redundant sequences, the identified putative trypsin protein

306 sequences were submitted to (https://

307 www.ncbi.nlm.nih.gov/Structure/bwrpsb/bwrpsb.cgi), Pfam and SMART (http://smart.embl-

308 heidelberg.de/) to confirm the conserved trypsin domain. All the non-redundant and high-

309 confidence genes were assigned as trypsin, named with abbreviated species names,

310 Cyanidioschyzon merolae (Cm), Thalassiosira pseudonana (Tp), Phaeodactylum tricornutum

311 (Pt), Thalassiosira oceanica (To), Fistulifera solaris $(F s)$, Fragilariopsis cylindrus $(F c)$,

312 Pseudo-nitzschia multiseries (Pm), Fugacium kawagutii (Fk), Chlamydomonas reinhardtii (Cr),

313 followed by Tryp standing for trypsin, then the order of their position on the

314 chromosome/scaffold/contig.

\section{Structural analysis of identified trypsin genes}

316 All of the high-confidence trypsin sequences we obtained were submitted to ExPASy

317 (http://web.expasy.org/protparam/) to calculate the number of amino acids, molecular weights

318 and theoretical isoelectric points (pI). The MEME program (version 4.11.2,

319 http://alternate.meme-suite.org/tools/meme) was used to identify the conserved motifs in the

320 trypsin sequences, with the following parameters: any number of repetitions, maximum of 10

321 misfits and an optimum motif width of 6 - 200 amino acid residues. The chromosomal positions

322 of the trypsin genes were acquired from the genome datasets of the species. MapChart

323 software ${ }^{35}$ was used for the mapping of trypsin genes' chromosomal positions and relative 324 distances.

325 Phylogenetic analysis of identified trypsin genes

326 OrthoFinder ${ }^{36,37}$ was used to identify the orthologous and paralogous groups among 9 marine phytoplankton species and the plant model Arabidopsis thaliana. All-versus-all BLASTP with an E value cutoff of $1 \mathrm{e}^{-05}$ were performed, orthologous and paralogous genes were clustered using OrthoFinder. Single-copy orthologous genes were extracted from the clustering results. 
330 To examine the grouping of the identified trypsin and trypsin-like genes, the deduced amino

331 acid sequences of these genes from the nine species of algae with sequenced genomes were

332 subjected to phylogenetic analysis. The alignment of the sequences was carried out using 333 ClustalW $^{38,39}$ and inspected manually for necessary correction. An unrooted Maximum

334 Likelihood phylogenetic tree was constructed using MEGA $\mathrm{X}^{40,41}$ software with bootstrap test 335 of 1000 times, based on a discrete Gamma distribution of evolutionary rate variations, which 336 was recommended by the results of Poisson correction model. The resulting tree file was 337 visualized with iTol (https://itol.embl.de).

Analyses of trypsin gene expression and correlation with environmental factors in the global ocean

The ambient nutrient conditions corresponding to trypsin expression data in the global ocean were extracted from PANGAEA (doi:10.1594/PANGAEA.836319) ${ }^{42}$. The following nine environmental nutrient parameters were chosen for correlation analysis: iron_5m $(\mathrm{Fe}, \mu \mathrm{mol} / \mathrm{l})$, ammonium_5m $\left(\mathrm{NH}_{4}^{+}, \mu \mathrm{mol} / \mathrm{l}\right), \mathrm{NO}_{2} \mathrm{NO}_{3}(\mu \mathrm{mol} / \mathrm{l}), \mathrm{NO}_{2}(\mu \mathrm{mol} / 1), \mathrm{NO}_{3}^{-}(\mu \mathrm{mol} / \mathrm{l}), \mathrm{PO}_{4}$

$344(\mu \mathrm{mol} / 1), \mathrm{Si}(\mu \mathrm{mol} / 1), \mathrm{NO}_{2} \_5 \mathrm{~m}(\mu \mathrm{mol} / 1), \mathrm{NO}_{3} \_5 \mathrm{~m}(\mu \mathrm{mol} / 1)$. The correlations of these environmental parameters with trypsin mRNA profiles were investigated using pairwise Spearman correlation analysis, with a "fdr"-adjusted $P$ value. Correlations were considered significant with a $P$ value $<0.05$ and an absolute coefficient $>30$.

Analysis of trypsin gene expression in diatoms and dinoflagellates in existing databases

The gene expression data of diatom trypsin genes (PtTryp for P. tricornutum and TpTryp for T. pseudonana trypsins) and dinoflagellate trypsin genes (FkTryp for F. kawagutii trypsin) were downloaded from the Diatom EST Database (http://www.diatomics.biologie.ens.fr/EST3/) and SAGER Database (http://sampgr.org.cn/index.php), respectively. The gene expression data included a range of environmental conditions, as detailed in websites. The counts of ESTs for PtTryp and TpTryp and TPM value for FkTryp were used to analyze the expression pattern. The results visualized using TBtools ${ }^{43}$. 
357 We wish to thank Xin Lin, Ling Li, Chentao Guo of Marine EcoGenomics Laboratory (Xiamen

358 University, China) for technical advice and assistance. We also thank Jianwei Chen (BGI

359 Genomics Co., Ltd., China) for his assistance with trypsin orthologous and paralogous analysis.

360 This work was supported by the National Natural Sciences Foundation of China (grant number

36141906123 to YY), the Open Fund of CAS Key Laboratory of Marine Ecology and

362 Environmental Sciences, Institute of Oceanology, Chinese Academy of Sciences (grant number

363 KLMEES202006 to YY), the Marine S\&T Fund of Shandong Province for Pilot National

364 Laboratory for Marine Science and Technology (Qingdao) (grant number 2018SDKJ0406-3 to

365 SL), the MEL Internal Programs of the State Key Laboratory of Marine Environmental Science,

366 Xiamen University (grant number 202105 to SL). We wish to thank the program of Excellent

367 Postdoctoral Allowance for Marine Science from the College of Ocean and Earth Sciences

368 (Xiamen University, China).

\section{Author contributions}

370 YY and SL conceived and designed the research. YY and XS performed the experiments and 371 data analysis. YY and SL wrote the manuscript.

\section{$372 \quad$ References}

373 1. Kühne, W. Ueber die. Verdauung der eiweisstoffe durch den pankreassaft. Archiv für 374 pathologische anatomie und physiologie und für klinische medicin 39, 130-174 (1867).

375 2. Rungruangsak-Torrissen, K. \& Male, R. in Seafood enzymes, utilization and influence on 376 postharvest seafood quality (eds N.F. Haard \& B.K. Simpson) Ch. Trypsin isozymes: 377 development, digestion and structure, 215-269 (2000).

378 3. Chen, J. M. et al. Evolution of trypsinogen activation peptides. Molecular biology and 379 evolution 20, 1767-1777 (2003).

380 4. Rojas, A. \& Doolittle, R. F. The occurrence of type S1A serine proteases in sponge and

5. Querino Lima Afonso, M., da Fonseca, N. J., Jr., de Oliveira, L. C., Lobo, F. P. \& Bleicher, 383 L. Coevolved positions represent key functional properties in the trypsin-like serine 
proteases protein family. J Chem Inf Model 60, 1060-1068 (2020).

6. Stryer, L. in Biochemistry (ed Lubert Stryer) Ch. 4.2, 233-259 (W.H. Freeman, 1988).

7. Rypniewski, W. R., Perrakis, A., Vorgias, C. E. \& Wilson, K. S. Evolutionary divergence and conservation of trypsin. Protein Eng 7, 57-64 (1994).

8. Rungruangsak-Torrissen, K., Moss, R., Andresen, L. H., Berg, A. \& Waagbo, R. Different expressions of trypsin and chymotrypsin in relation to growth in Atlantic salmon (Salmo salar L.). Fish Physiol Biochem 32, 7-23 (2006).

9. Torrissen, K. R., Male, R. \& Naevdal, G. Trypsin isozymes in Atlantic salmon, Salmo salar L.: studies of heredity, egg quality and effect on growth of three different populations.

10. Zhang, Y. Q. et al. Metatranscriptomic signatures associated with phytoplankton regime Aquaculture Research 24, 407-415 (1993). shift from diatom dominance to a dinoflagellate bloom. Frontiers in microbiology 10 (2019).

11. Gu, Z. L., Cavalcanti, A., Chen, F. C., Bouman, P. \& Li, W. H. Extent of gene duplication in the genomes of Drosophila, nematode, and yeast. Molecular biology and evolution 19, 256-262 (2002).

12. Maheswari, U., Mock, T., Armbrust, E. V. \& Bowler, C. Update of the diatom EST database: a new tool for digital transcriptomics. Nucleic Acids Research 37, D1001-D1005 (2009).

13. Li, T. C. et al. RNA-seq profiling of Fugacium kawagutii reveals strong responses in metabolic processes and symbiosis potential to deficiencies of iron and other trace metals. Sci Total Environ 705 (2020).

14. Eisen, M. B., Spellman, P. T., Brown, P. O. \& Botstein, D. Cluster analysis and display of genome-wide expression patterns. Proceedings of the National Academy of Sciences of the United States of America 95, 14863-14868 (1998).

15. Neurath, H. Evolution of proteolytic enzymes. Science 224, 350-357 (1984).

16. Hooper, N. M. Proteolytic enzymes: A practical approach Edited by R J Beynon and J S Bond. pp 259. IRL Press at Oxford University Press, Oxford. 1989. Biochemical Education 18, 55-55 (1990).

17. Davis, C. A., Riddell, D. C., Higgins, M. J., Holden, J. J. \& White, B. N. A gene family in Drosophila melanogaster coding for trypsin-like enzymes. Nucleic Acids Res 13, 6605- 
6619 (1985).

18. Muller, H. M., Crampton, J. M., della Torre, A., Sinden, R. \& Crisanti, A. Members of a trypsin gene family in Anopheles gambiae are induced in the gut by blood meal. EMBO J 12, 2891-2900 (1993).

19. Stevenson, B. J., Hagenbuchle, O. \& Wellauer, P. K. Sequence organization and transcriptional regulation of the mouse Elastase-Ii and trypsin genes. Nucleic Acids Research 14, 8307-8330 (1986).

20. Roach, J. C., Wang, K., Gan, L. \& Hood, L. The molecular evolution of the vertebrate trypsinogens. Journal of molecular evolution 45, 640-652 (1997).

21. Arenas, A. F., Osorio-Mendez, J. F., Gutierrez, A. J. \& Gomez-Marin, J. E. Genome-wide survey and evolutionary analysis of trypsin proteases in apicomplexan parasites. Genomics Proteomics Bioinformatics 8, 103-112 (2010).

22. Lespinet, O., Wolf, Y. I., Koonin, E. V. \& Aravind, L. The role of lineage-specific gene family expansion in the evolution of eukaryotes. Genome Res 12, 1048-1059 (2002).

23. Rowen, L., Koop, B. F. \& Hood, L. The complete 685-kilobase DNA sequence of the human beta T cell receptor locus. Science 272, 1755-1762 (1996).

24. Lin, H. et al. Genome-wide identification and expression profiling of serine proteases and homologs in the diamondback moth, Plutella xylostella (L.). BMC Genomics 16, 1054 (2015).

25. Wu, D. D., Wang, G. D., Irwin, D. M. \& Zhang, Y. P. A profound role for the expansion of trypsin-like serine protease family in the evolution of hematophagy in mosquito. Molecular biology and evolution 26, 2333-2341 (2009).

26. Zou, Z., Lopez, D. L., Kanost, M. R., Evans, J. D. \& Jiang, H. Comparative analysis of serine protease-related genes in the honey bee genome: possible involvement in embryonic development and innate immunity. Insect Mol Biol 15, 603-614 (2006).

27. Hellman, L. \& Thorpe, M. Granule proteases of hematopoietic cells, a family of versatile inflammatory mediators - an update on their cleavage specificity, in vivo substrates, and evolution. Biol Chem 395, 15-49 (2014).

28. Schuhmann, H. \& Adamska, I. Deg proteases and their role in protein quality control and processing in different subcellular compartments of the plant cell. Physiol Plantarum 145, 
224-234 (2012).

29. Schuhmann, H., Huesgen, P. F. \& Adamska, I. The family of Deg/HtrA proteases in plants. BMC Plant Biol 12, 52 (2012).

30. Zhang, Y.Q. et al. Metatranscriptomic signatures associated with phytoplankton regime shift from diatom dominance to a dinoflagellate bloom. Frontiers in microbiology 10, 590, (2019).

31. Muhlia-Almazan, A., Sanchez-Paz, A. \& Garcia-Carreno, F. L. Invertebrate trypsins: a review. J Comp Physiol B 178, 655-672 (2008).

32. Carradec, Q. et al. A global ocean atlas of eukaryotic genes. Nat Commun 9, 373 (2018).

33. Yu, L. et al. SAGER: a database of Symbiodiniaceae and Algal Genomic Resource. Database (Oxford) 2020, (2020).

34. Villar, E. et al. The Ocean Gene Atlas: exploring the biogeography of plankton genes online. Nucleic Acids Res 46, 289-295 (2018).

35. Voorrips, R. E. MapChart: Software for the graphical presentation of linkage maps and QTLs. J Hered 93, 77-78 (2002).

36. Emms, D. M. \& Kelly, S. OrthoFinder: solving fundamental biases in whole genome comparisons dramatically improves orthogroup inference accuracy. Genome Biol 16, 157 (2015).

37. Emms, D. M. \& Kelly, S. OrthoFinder: phylogenetic orthology inference for comparative genomics. Genome Biol 20, 238 (2019).

38. Thompson, J. D., Higgins, D. G. \& Gibson, T. J. CLUSTAL W: improving the sensitivity of progressive multiple sequence alignment through sequence weighting, position-specific gap penalties and weight matrix choice. Nucleic Acids Res 22, 4673-4680, (1994).

39. Larkin, M. A. et al. Clustal W and Clustal X version 2.0. Bioinformatics 23, 2947-2948 (2007).

40. Kumar, S., Stecher, G., Li, M., Knyaz, C. \& Tamura, K. MEGA X: molecular evolutionary genetics analysis across computing platforms. Molecular biology and evolution 35, 1547 1549 (2018).

41. Stecher, G., Tamura, K. \& Kumar, S. Molecular Evolutionary Genetics Analysis (MEGA) for macOS. Molecular biology and evolution 37, 1237-1239 (2020). 
474 42. Pesant, S. et al. Open science resources for the discovery and analysis of Tara Oceans data. 475 Sci Data 2, 150023 (2015).

476 43. Chen, C., Xia, R., Chen, H. \& He, Y. TBtools, a Toolkit for Biologists integrating various 477 HTS-data handling tools with a user-friendly interface. bioRxiv, 289660, 478 doi:10.1101/289660 (2018).

479 
Fig. 1 Geographic and taxonomic distribution of trypsin and trypsin-like homologs detected by mining the Tara Oceans eukaryote unigene catalog and metatranscriptomes (MATOUv1+metaT) datasets in the OGA webserver. a, Geographic distribution and abundance of trypsin homologs in eukaryotes in surface layer (SRF) and deep chlorophyll maximum layer (DCM). b, Geographic distribution and abundance of trypsin-like homologs in eukaryotes in SRF and DCM. c, Taxonomic distribution of all the detected trypsin homologs in eukaryotes. d, Taxonomic distribution of all the detected trypsin-like homologs in eukaryotes. The size of the filled circles shown in $(a, b)$ are proportional to the mRNA abundance of the hits in one location compared to the total number of hits integrated from the different sampled depths, and colors represent the size fractionation range.

Fig. 2 Evolutionary dynamics of genome size, abundance of coding genes, and abundance of trypsin genes among selected algae and the land plant model $\boldsymbol{A}$. thaliana. The phylogenetic tree was constructed based on NCBI taxonomy and TIMETREE database. Lineage divergence time is indicated at each branch point; Mya, million years ago. The number in trypsin column represents the number of identified trypsin genes, and the number in parenthesis indicates the number of duplication gene pairs.

Fig. 3 Phylogenetic relationship and architectural profiles of conserved domain and conserved motif of trypsin genes in algae. Located in the core is the phylogenetic tree of 291 trypsin and trypsin-like proteins from all nine algal species, which groups these proteins into two clusters (I, trypsin; II, trypsin-like). The unrooted maximum likelihood phylogenetic tree was constructed with MEGA7, and the bootstrap test replicate was set as 1000 times. $\mathrm{Cm}$ : Cyanidioschyzon merolae, Tp: Thalassiosira pseudonana, Pt: Phaeodactylum tricornutum, To: Thalassiosira oceanica, Fs: Fistulifera solaris, Fc: Fragilariopsis cylindrus, Pm: Pseudonitzschia multiseries, Fk: Fugacium kawagutii, Cr: Chlamydomonas reinhardtii. Inner cycle depicts trypsin genes from different lineages of algae corresponding to branches of the phylogenetic tree, each lineage in a unique color: red, diatoms; purple, dinoflagellate; blue, green alga; yellow, red alga. Middle circle: green, deep pink and yellow rectangles represent 
trypsin, trypsin_like and PDZ conserved domains, respectively. Outer circle illustrates different organizations of ten putative motifs, each in a different color of rhombus. UN: unknown, tryp: trypsin. For details of motifs refer to Extended Data Table 2.

511 Fig. 4 Number and location of duplicated trypsin gene pairs. a, Distribution of tandem

512 duplicated trypsin genes on $C$. reinhardtii genome. b, Duplicated trypsin gene pairs in $T$.

513 oceanica. c, Duplicated trypsin gene pairs in F. solaris. d, Distribution of segmentally

514 duplicated trypsin genes on F. solaris genome. Duplicated trypsin genes were mapped to their

515 respective locus in the genome in a circular diagram using Circos. Chromosomal or scaffold 516 segments are indicated by different colors (outer track). Duplicated trypsin gene pairs linked

517 with green or red lines (inside of circle). Grey lines indicate collinear blocks in whole genome.

518 Fig. 5 Phylogenetic relationships of paralogs and orthologs trypsin genes between

519 different marine phytoplankton. The phylogenetic tree was constructed on 234 trypsin genes

520 that identified as paralogs and orthologs groups from 307 trypsin genes by OrthoFinder. Based

521 on the paralogs and orthologs groups, the phylogenetic tree was manually defined into 9 clades.

522 All the branches within clades have been collapsed for simplicity and the number of sequences

523 corresponding to each selected species. Sequences from the same species within the same clade

524 were considered as paralogs groups, and sequences from different species were considered as

525 orthologs groups. Combining trypsin phylogenetic tree and the simplified phylogenies of

526 selected species (upper tree), we observe a variable gene number in each clade among the 527 species, indicating an evolutionary scenario characterized by several gene duplication and loss 528 events.

529 Fig. 6 Sample distribution of identified trypsin transcripts and pairwise Spearman 530 correlation between trypsin subfamily mRNA level and environmental parameters. a, The 531 trypsins that contain trypsin domain (Pfam ID PF00089) showing little correlation with 532 environmental factors. b, The trypsin-like genes that contain trypsin-like peptidase domain 533 (Pfam ID PF13365) showing extensive correlations with environmental factors. The 534 correlations were run for each size class and $\mathrm{p}$ values were adjusted based false discovery rate 535 (FDR). Only significant correlations $(p$-value $<0.05)$ are shown. Data of mRNA level and 536 environmental parameters were from the 65 Tara Oceans sampling stations at which seawater 
samples and environmental data were collected from surface (SRF) and deep chlorophyll maximum depths (DCM). The width of line that links from gene to sample is proportional to the ration of trypsin mRNA abundance distribution in different samples. The length of factor bar (on the right) is proportional to the effect of environmental factors. The $+/$ - sign represents positive/negative correlation.

Fig. 7 Expression profiles of trypsin genes of $P$. tricornutum, $T$. pseudonana and $F$. kawagutii across different conditions. ESTs for $P$. tricornutum and $T$. pseudonana trypsin genes, TPM values for F. kawagutii trypsin genes. ESTs and TPM value of trypsin genes were transformed by $\log _{2}$ and the heatmap was constructed by Tbtools software. a, Hierarchical clustering of expression patterns of $P$. tricornutum trypsin genes in 16 different growth conditions. b, Hierarchical clustering of expression patterns of T. pseudonana trypsin genes in seven growth conditions. c, Expression patterns of $F$. kawagutii trypsin genes under six growth conditions. Abbreviations of library names, Pt2DB: OS, original 12000 standard; AA, ammonium adapted; FL, iron limited; SP, silica plus; SM, silica minus; NR, nitrate replete; NS, nitrate starved; UA, urea adapted; LD, low decadienal treated; HD, high decadienal treated; OM, oval morphotype; TM, triradiate morphotype; TA, tropical accessions; BL, blue light; $\mathrm{C} 1$, high $\mathrm{CO}_{2}$ for1 d; $\mathrm{C} 4$, high $\mathrm{CO}_{2}$ for $4 \mathrm{~d}$. Tp2DB: FL, iron limited cells (-Fe); NL, nitrate limited cells (-NO3); NP, nitrate plus; TL, temperature limited; CL, carbon dioxide limited cells (-CO2); OL, old library; SL, silicate limited cells (-Si). SAGER: -Cu, without $\mathrm{Cu}$; -Mn, without $\mathrm{Mn}$; $\mathrm{Ni}$, without $\mathrm{Ni} ;+1 / 5 \mathrm{Zn}: 2 \mathrm{nM}, 1 / 5$ of its normal concentration; +1/5 Fe, $50 \mathrm{nM}, 1 / 5$ of its normal concentration; NR, nitrate replete. The gradient of color shows the level of expression, with the darker colors representing higher expression.

\section{Extended Data}

Extended Data Table $1 \mathrm{Ka} / \mathrm{Ks}$ values of duplicated trypsin gene pairs.

561 Extended Data Table 2 List of the putative motifs of 291 alga trypsin proteins.

562 Extended Data Fig. 1 Chromosome/scaffold location of trypsin genes. a, C. reinhardtii; b,

563 F. cylindrus; c, F. solaris; d, T. pseudonana; Chr: chromosome; The bar length represents the 
564 length of chromosome or scaffold. The trypsin location of other species that no showed here,

565 due to their absence of gene clusters or their genome assembly at contig level.

566 Extended Data Fig. 2 WebLogo consensus sequences for 10 most conserved motifs of algal 567 trypsins.

568 Supplementary File

569 Supplementary Table 1 Gene characteristics of algal trypsin genes. 
a

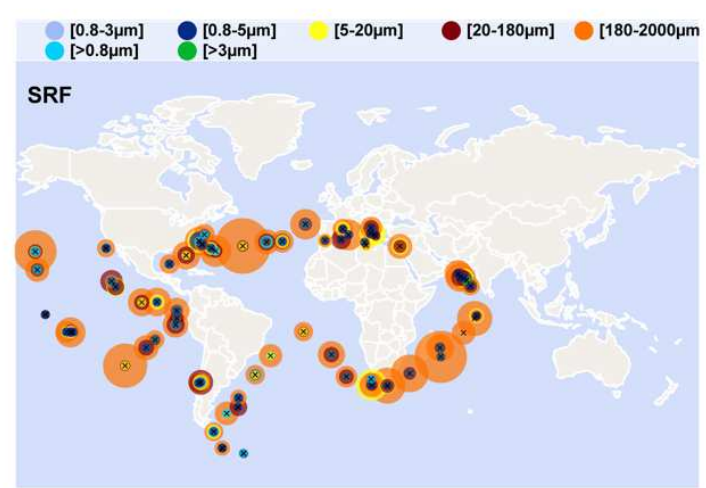

DCM

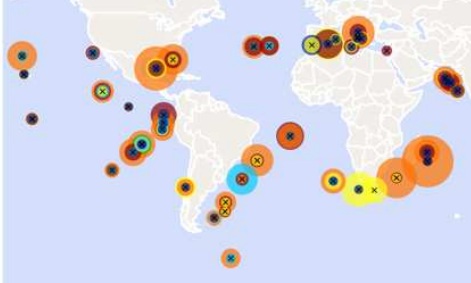

b

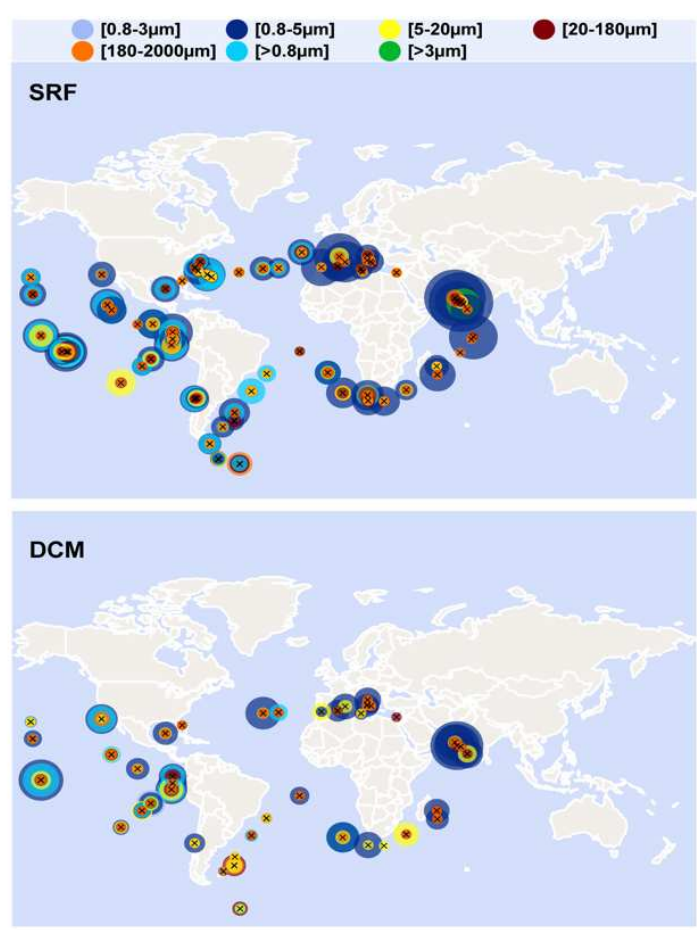

C

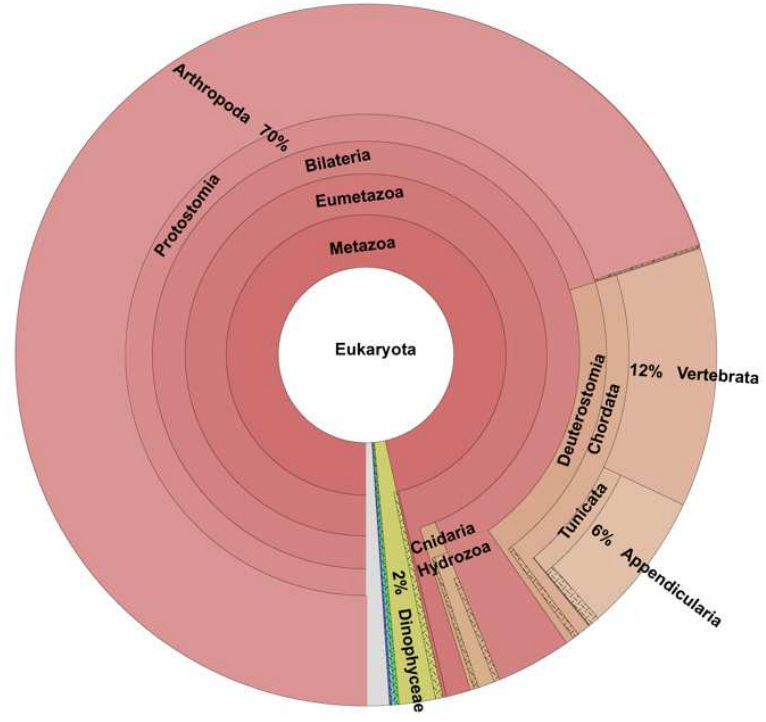

d

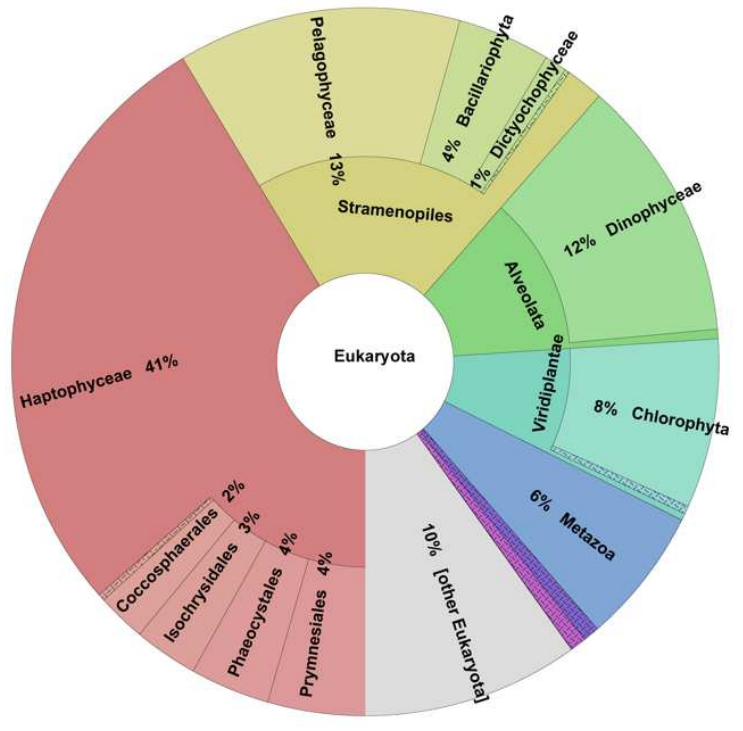




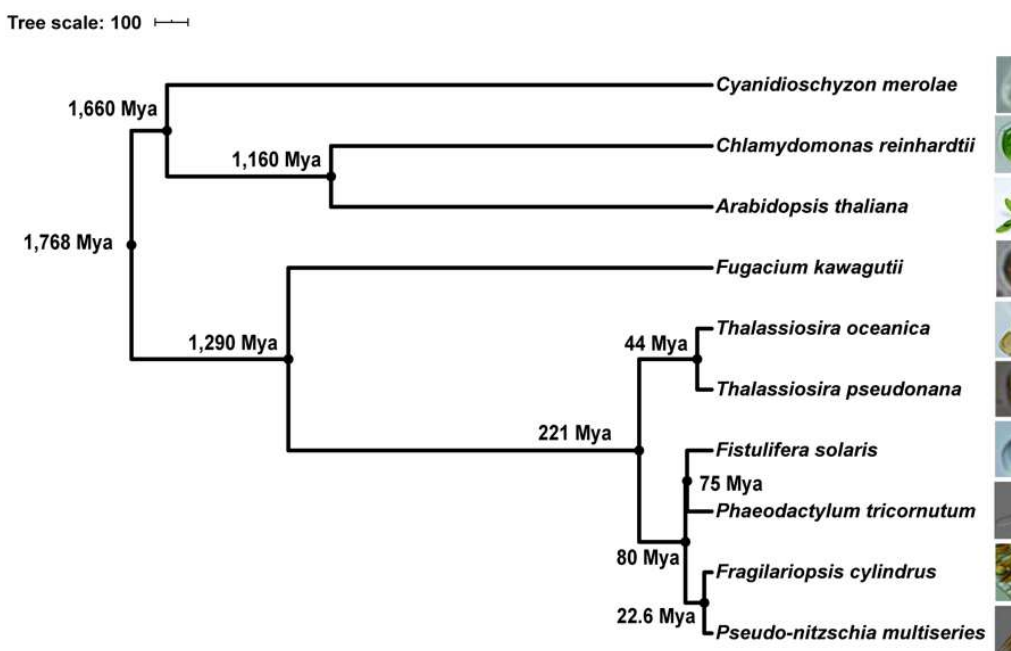

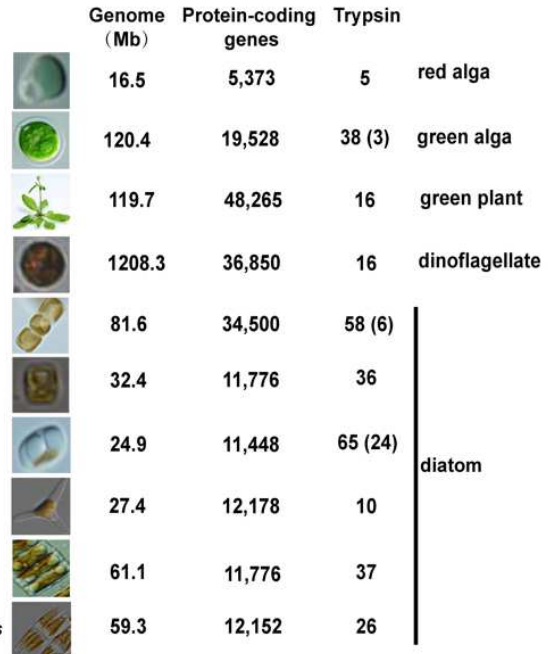

571

572 


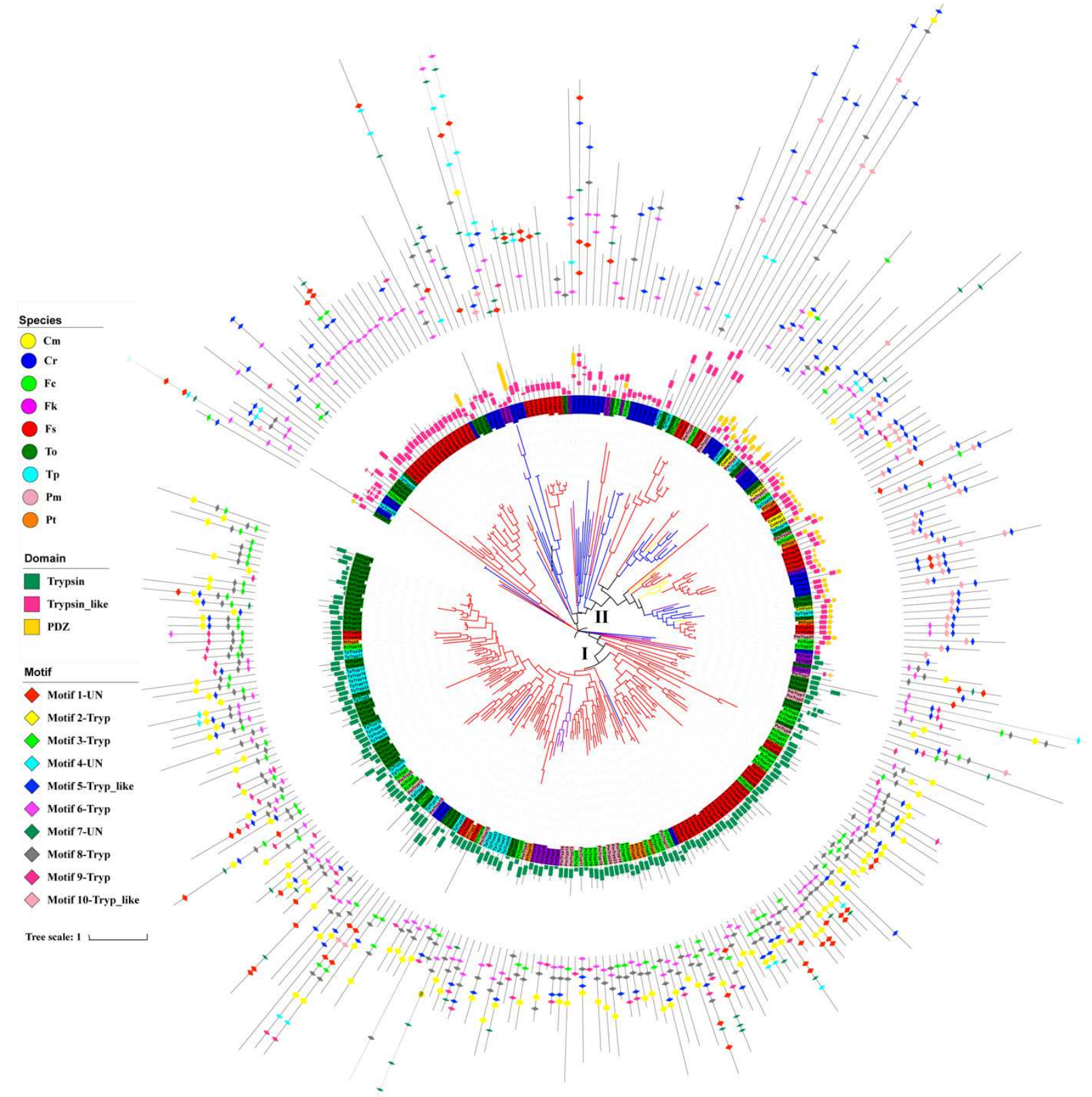



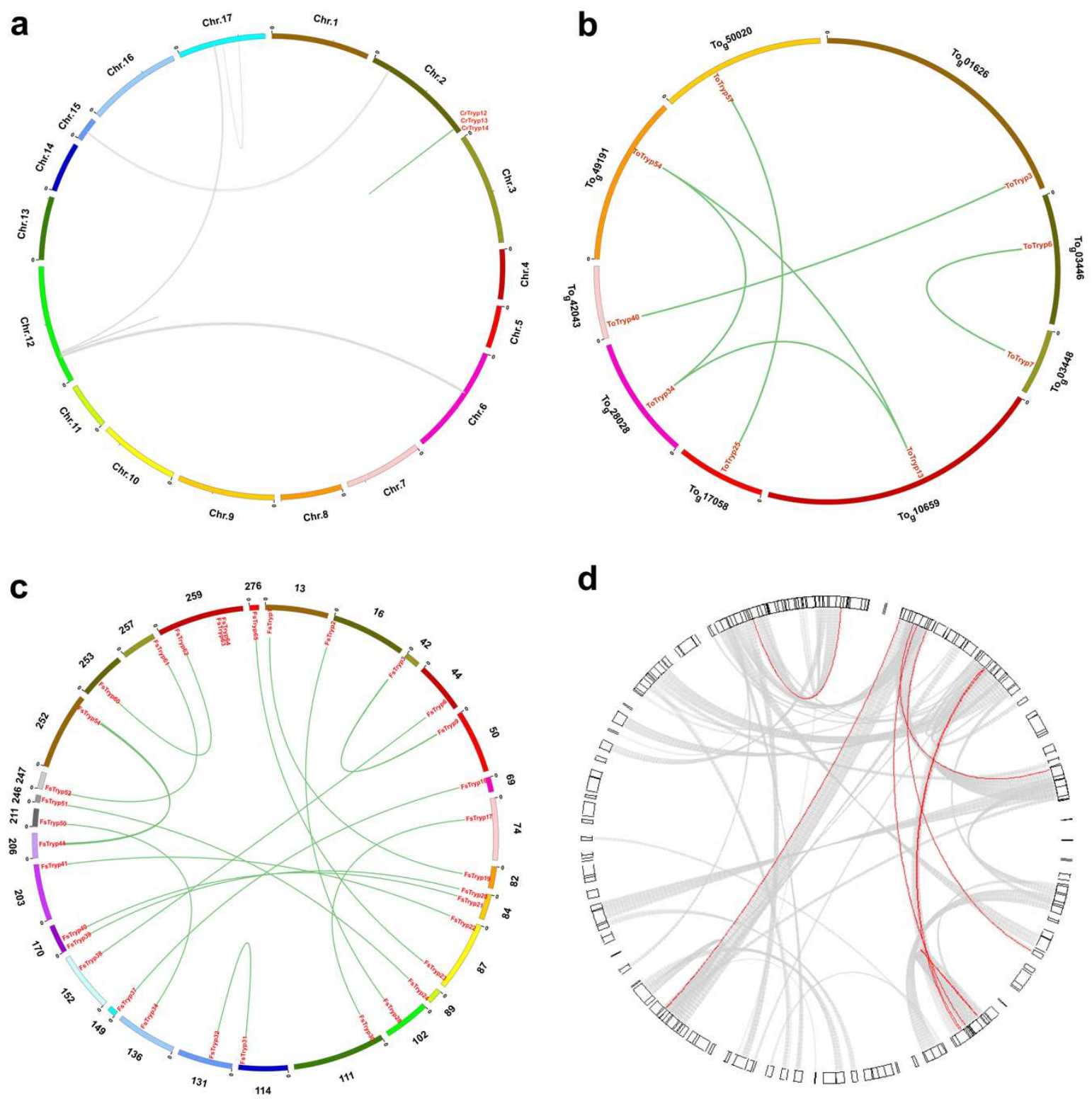


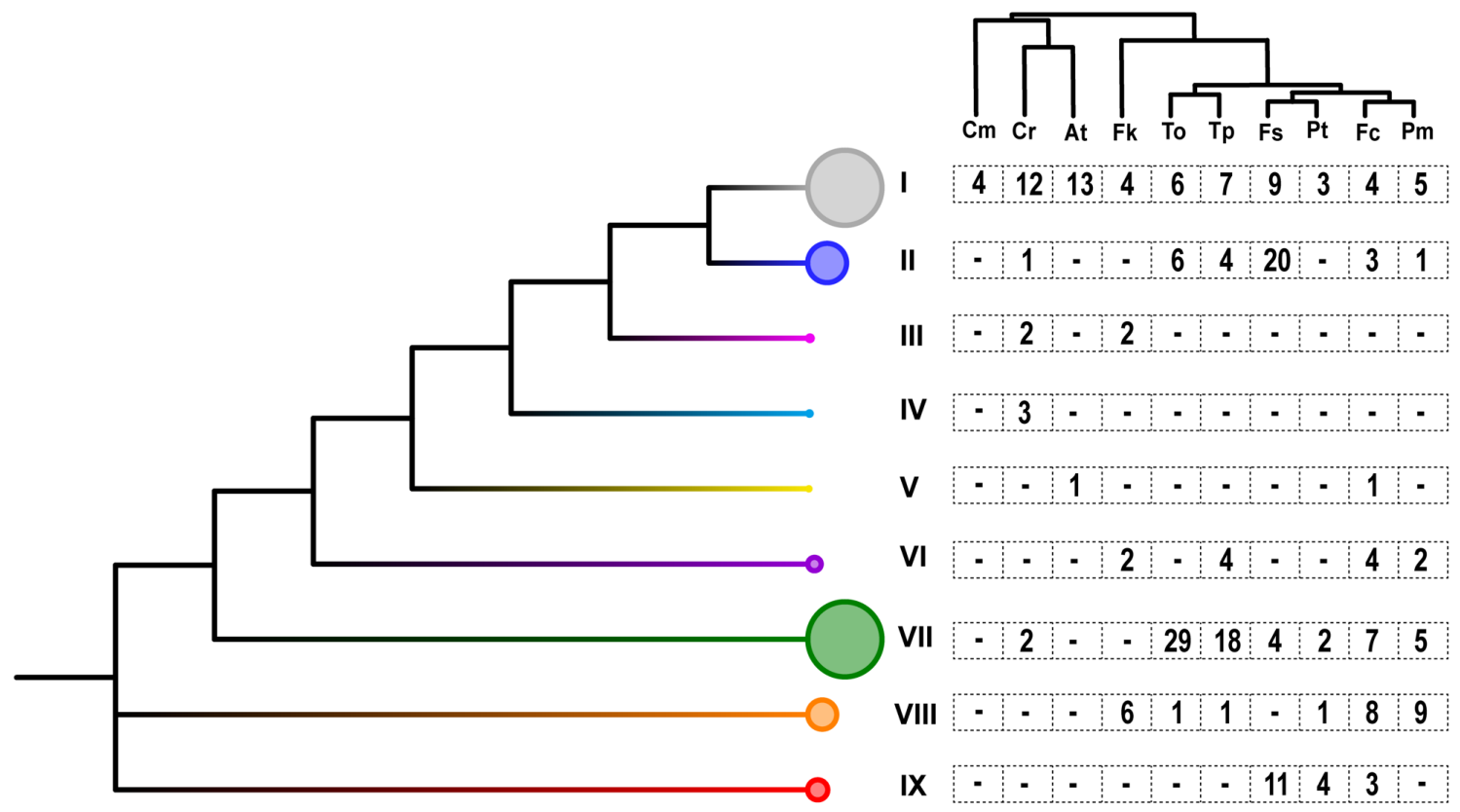

575

29 
a

Gene Sample

Factor Correlation

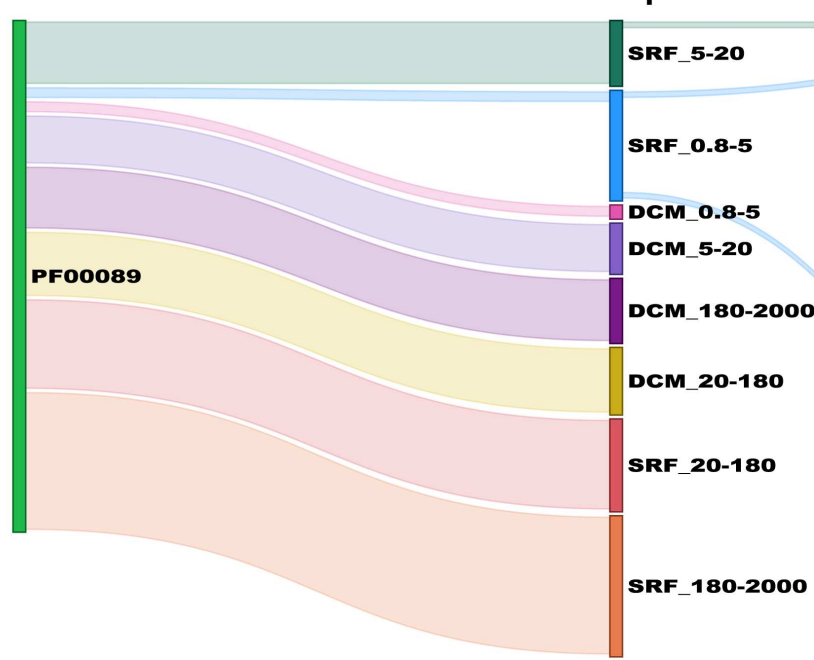

b

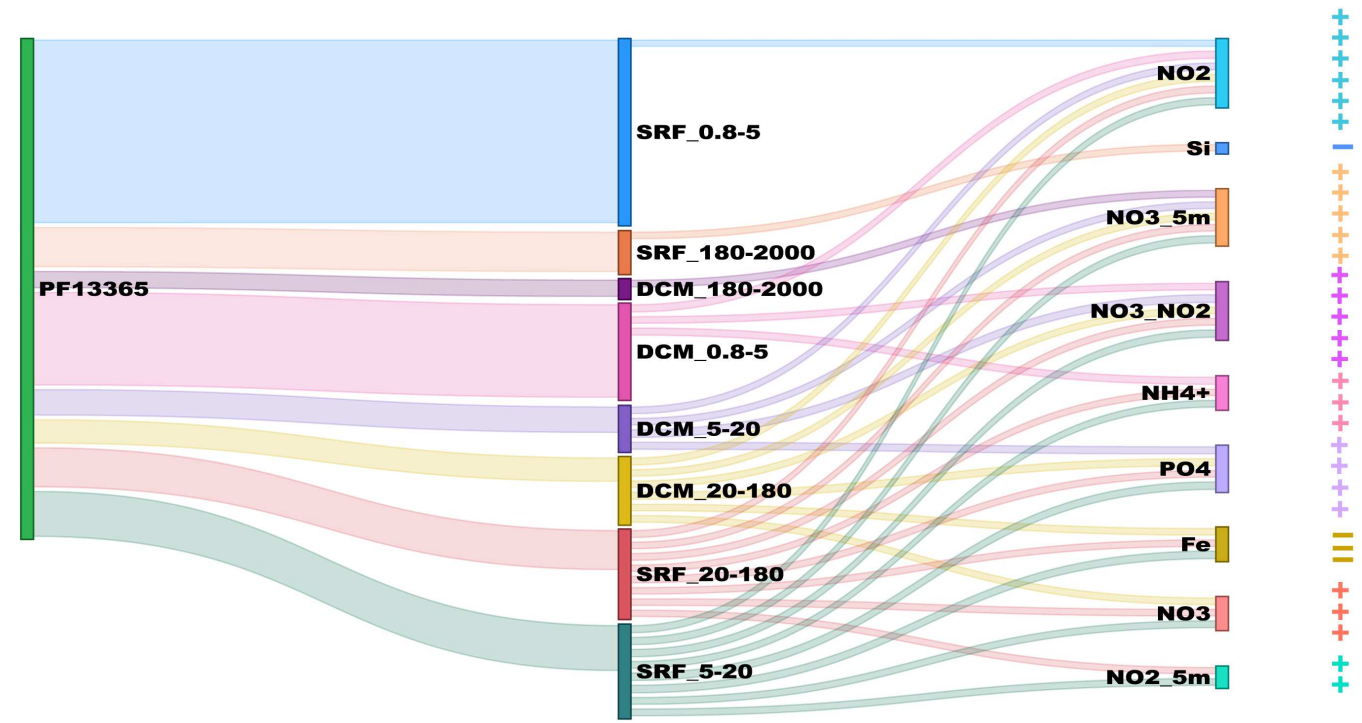


a
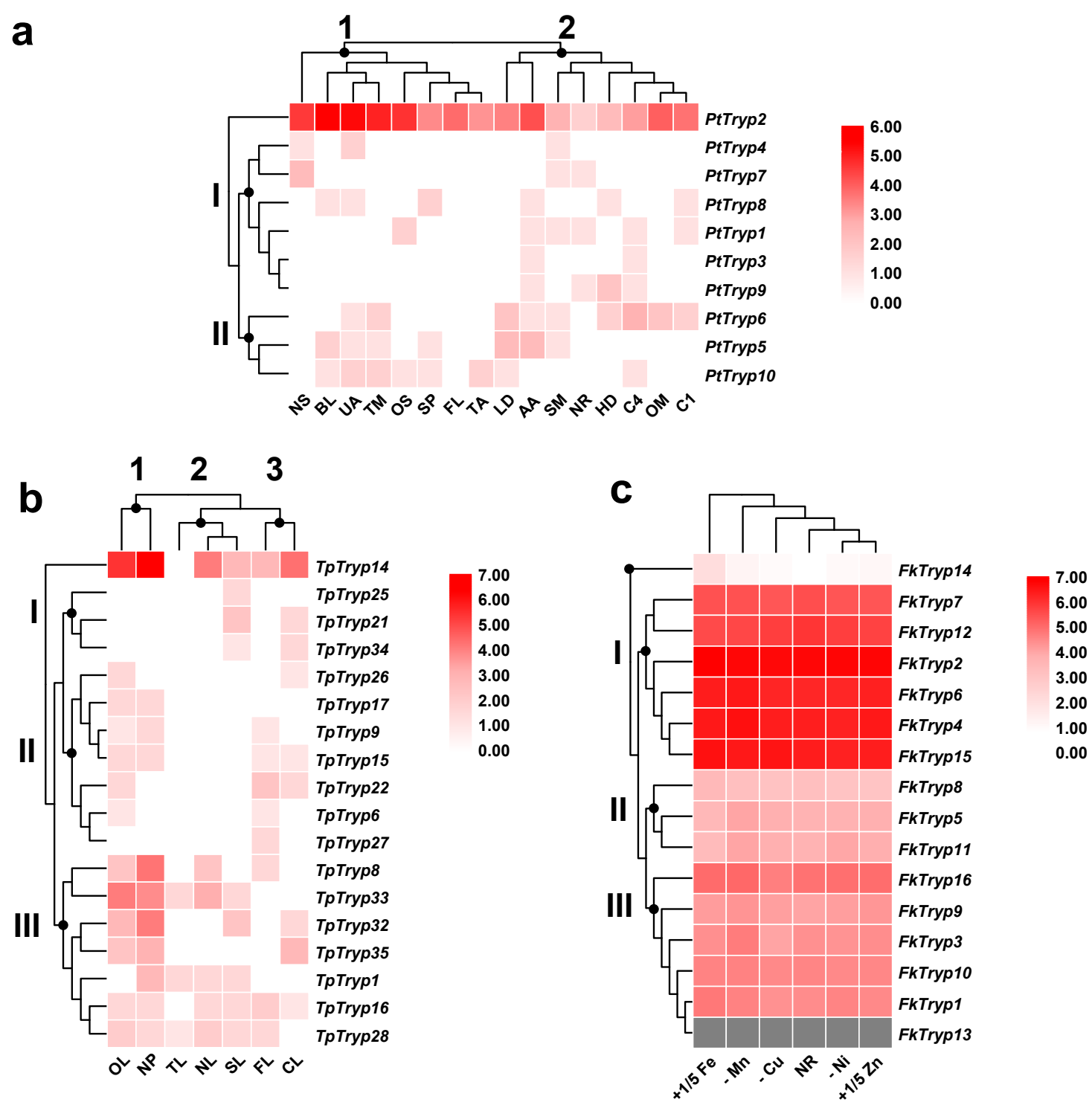


\section{Supplementary Files}

This is a list of supplementary files associated with this preprint. Click to download.

- ExtendedDataCB.pdf

- SupplementaryFile.xlsx 